\title{
CUIDADOS DEL RECIÉN NACIDO
} PREMATURO EN CASA

\section{Intervención educativa aplicando el modelo de autocuidado de Dorothea Orem}

Marina Higuera de Quintero*, Heidy Christiansen, Claudia Cifuentes, Diana Contreras, Paola Cubillos, Diana Gutiérrez, Yeimy Martinez, Edith Triana**

\section{Resumen}

Para el desarrollo de este estudio se contó con la evaluación preliminar de los conocimientos de un grupo de 20 madres cuyos hijos estuvieron hospitalizados en la unidad de recién nacidos del Hospital de San José durante el segundo semestre del 2003. Los criterios de inclusión fueron: madres cuyos hijos habían superado la fase crítica de la enfermedad, madres de recién nacidos con pesos entre 1.200 y 2.000 g y madres con edades entre 18 y 35 años. Posteriormente, durante el segundo semestre del 2004 se seleccionó un grupo de 20 madres con los mismos criterios de inclusión a quienes se les dictó un programa educativo sobre los cuidados del niño prematuro en casa. Una vez desarrollado el programa se hizo comparación entre los dos grupos. El presente estudio es de tipo comparativo transversal entre un grupo intervenido y uno no intervenido y tiene como objetivo evaluar la influencia de un programa educativo sobre los cuidados niño prematuro en casa, aplicando el modelo de autocuidado de Dorothea Orem .

La comparación entre los dos grupos se realizó aplicando una prueba de diferencia de proporciones con un nivel de significancia del 0.05. Los resultados obtenidos confirmaron la hipótesis planteada, por lo que se considera que el programa educativo fue para el grupo de madres significativo en cuanto a la obtención de conocimientos.

intervención educativa, recién nacido prematuro, autocuidado, conocimientos, grupo de madres.

\section{Introducción}

Los niños que han nacido prematuramente no solo presentan con más frecuencia problemas motores, psíquicos y sensoriales, también tienen problemas del crecimiento y problemas infecciosos. Estas circunstancias determinan que la demanda de los padres sea mayor y que en ocasiones, requieran intervenciones específicas respecto a los problemas que presentan.

Ninguna pareja está preparada para tener un hijo que nazca muy prematuramente y esto sin duda afec-

\footnotetext{
Investigadora principal. Secretaria Académica Fundación Universitaria de Ciencias de la Salud, Profesora Asociada. Magister en Enfermería pediátrica.

** Coinvestigadoras. Estudiantes de VII Semestre Facultad de Enfermería, Fundación Universitaria de Ciencias de la Salud.
}

ta a las relaciones padres - hijos, no sólo durante el ingreso del niño. La incertidumbre sobre la evolución es grande y condiciona en ocasiones, actitudes que dificultan la plena aceptación del hijo. La enfermera, como profesional más próximo a la familia, puede ayudar, también en ese sentido, a identificar estos problemas y proporcionar información precisa que disminuya la incertidumbre.

Los padres desde el primer contacto con la prematurez, inician un camino salpicado de angustia, temor e incertidumbre, que muchas veces se prolonga más allá de la hospitalización del niño. ${ }^{1,2}$ El cuidado hacia el hijo prematuro es ambivalente, lo maltratan, abandonan o lo sobreprotegen. Se deteriora la imagen de sí mismo, la relación de pareja y la interacción con los otros hijos, lo que muchas veces culmina en la ruptura matrimonial. 
En 1990, Wyly y Allen ${ }^{3}$ describieron cómo los padres de prematuros atraviesan distintas etapas afectivas a lo largo de sus vidas. Probablemente, algunas se inician antes del nacimiento y otras se manifiestan a lo largo de la hospitalización.

Este trabajo propone el establecimiento de un programa educativo dirigido a las madres con el objetivo de permitirles acceder a un conocimiento sobre los cuidados del niño prematuro en casa. Cuidados que abarcan aspectos físicos como la alimentación, posición, higiene y los aspectos emocionales. El principal propósito de este trabajo fue el desarrollo de una estrategia de intervención educativa para incrementar los conocimientos de las madres sobre los cuidados de los niños prematuros. Esta intervención les permitirá a las madres adquirir de forma práctica, confiable y funcional un conocimiento que les lleve a tener una visión clara de la importancia de su papel en la crianza y que les brinde herramientas para poder ayudar a sus hijos a lograr un sano desarrollo.

Se espera que la formación de madres repercutirá no solo en el bienestar social y emocional de los niños, sino también en facilitarles una mejor disposición y preparación necesarias para el aprendizaje escolar. Por otra parte es de esperar que se desarrolle una mayor comprensión de las madres hacia sus hijos y una mejor forma de relacionarse con ellos, actitud que se refleja en un sentimiento de alta satisfacción de su rol parental.

\section{Método}

\section{- TIPO DE ESTUDIO}

Estudio comparativo de corte transversal entre una población no capacitada y una capacitada.

\section{- POBLACIÓN Y MUESTRA}

La población del estudio fue conformada por las madres de los niños prematuros que se encontraban hospitalizados en la unidad de recién nacidos del Hospital de San José durante los meses de agosto y septiembre de 2004
La muestra fue conformada por 20 madres que cumplieron con los criterios de inclusión. Este número se estableció teniendo en cuenta que fue determinado por el grupo de investigadores de la primera parte de este estudio, quienes para definir el tamaño de la muestra revisaron los archivos estadísticos de los niños prematuros hospitalizados en la unidad de recién nacidos del Hospital de San José desde el mes de enero hasta el mes de julio de 2.003. Ellos encontraron que mensualmente se hospitalizaron entre 13 y 20 recién nacidos cuyas madres e hijos reunían las características exigidas para el estudio.

\section{- CRITERIOS DE INCLUSIÓN:}

- Madres cuyos hijos se encuentren hospitalizados en la Unidad de recién nacidos del Hospital de San José .

- Madres de niños prematuros con un peso entre 1.200 y $2.000 \mathrm{~g}$ independiente de la edad gestacional y que hayan superado la fase crítica.

- Madres con edades entre 18 y 35 años.

- Madres que no hayan tenido antecedentes de hijos prematuros.

- Madres que tengan por lo menos primaria completa y sin formación profesional.

\section{- CRITERIOS DE EXCLUSIÓN:}

- Madres que no deseen participar en el estudio

- Madres con conocimientos sobre el manejo de niños prematuros .

\section{Intervención}

Después de que las madres recibieron la intervención educativa se les aplicó la encuesta CAP, que el grupo investigador de la primera parte de este estudio utilizó para identificar los conocimientos actitudes y prácticas de las madres sobre los cuidados del niño prematuro en casa. El instrumento se realizó, en su mayoría, con preguntas de selección múltiple y algunas dicotómicas; cada formulario contiene un instructivo que fue manejado por las investigadoras a fin de facilitar la claridad de la prueba y su contenido. 


\section{Resultados}

Como se observa en la figura en el "pretest" sólo entre 7 y 12 madres conocían el programa madre canguro, no había claridad sobre el objetivo, vestido y posición canguro. En cuanto al objetivo, pensaban que solo era para proporcionar calor al recién nacido y para aumento de peso; las madres de la población de estudio desconocen la importancia del contacto piel a piel como una necesidad básica del recién nacido, así mismo desconocían que la posición vertical o levantada es la adecuada para evitar broncoaspiración y apneas en el recién nacido. En el "postest" la totalidad de la muestra alcanzó el objetivo de la educación siendo esto de gran importancia para afianzar el vínculo madre- hijo, y evitar complicaciones en casa (Figura 1).

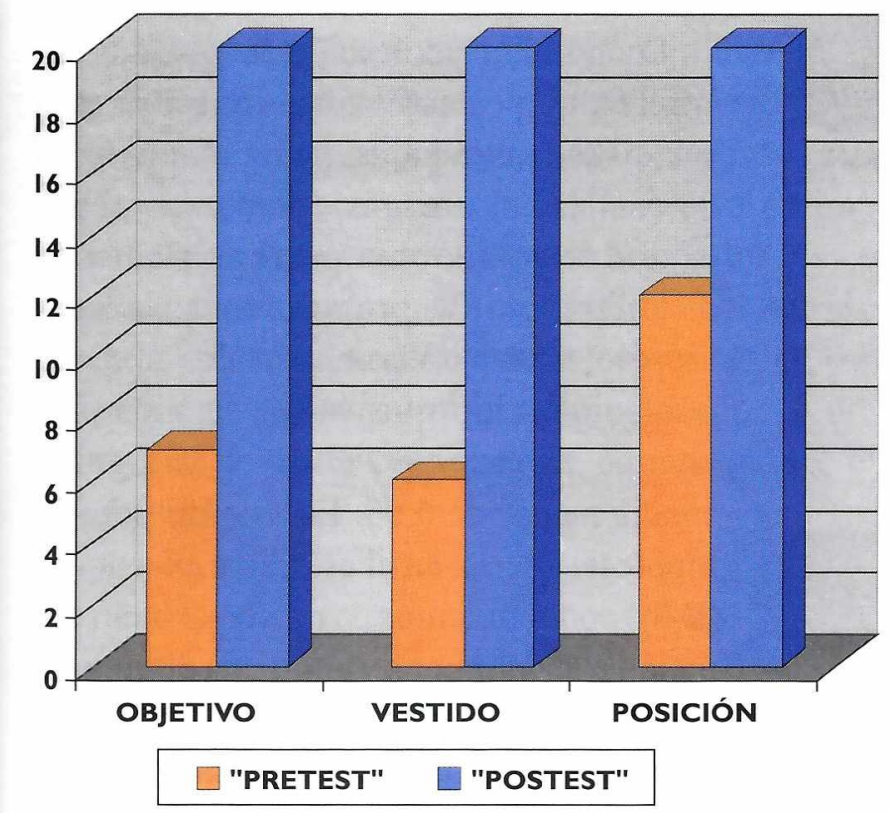

Figura I. Distribución de frecuencias de los conocimientos de las madres acerca del programa madre canguro antes y después de la intervención educativa.

En la figura 2 se observa que en el "pretest" algunas de las madres desconocían que al recién nacido no se le debe bañar, solo se debe limpiar diariamente con aceite para evitar hipotermia y disminución de las pérdidas insensibles. El conocimiento necesario para la realización de la higiene del recién nacido es de gran importancia ya que realizarlo de una manera inadecuada estaría sometiendo al niño a pérdida de peso lo cual estaría invalidando uno de los principios del programa madre canguro. En el "postest" la totalidad de la muestra obtuvo los conocimientos acerca de los temas (Figura 2).

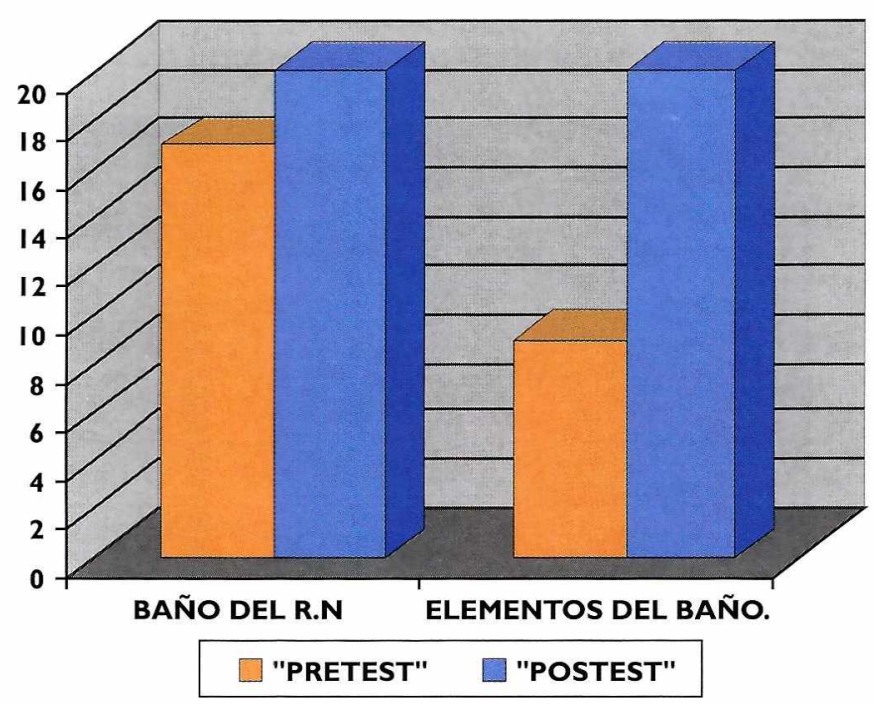

Figura 2. Distribución de frecuencias sobre los conocimientos de las madres acerca de la higiene del recién nacido antes y después de la intervención educativa.

Con respecto a la alimentación del niño prematuro es de gran importancia que las madres tengan conocimientos claros sobre el tipo, frecuencia, duración y conservación de la leche ya que al egreso del hospital ellas serán las encargadas de alimentar a sus hijos. Dentro de los resultados encontrados en el "pretest", entre 12 y 14 madres desconocían estos aspectos importantes acerca de la alimentación del niño prematuro. En el "postest" los resultados mostraron que la totalidad de las madres estaban preparadas para alimentar adecuadamente a sus hijos en casa (Figura 3).

Con relación a los controles médicos una vez el niño sea dado de alta de hospital, se valoraron los conocimientos de las madres sobre la asistencia a la primera consulta, primera toma de peso y aumento de peso diario. En el "pretest" sólo entre 2 y 10 madres conocían que a su hijo lo deben llevar a consulta y pesarlo al día siguiente después de salir del hospital, así mismo que el niño debe aumentar de peso mínimo 20 gramos diarios. En el "postest" 19 de las madres tuvieron claridad en estos aspectos (Figura 4). 
Además de los anteriores resultados el estudio mostró que conocer el tratamiento que se le esta dando a los recién nacidos permite disminuir los estados de ansiedad que afectan a las madres por no saber que le hacen a sus hijos dentro de la uni- dad de recién nacidos; es por esto que al evaluar el "pretest" se encontró que 15 madres conocían el tratamiento que estaban recibiendo sus hijos y en el "postest" la totalidad de la muestra obtuvo los conocimientos.

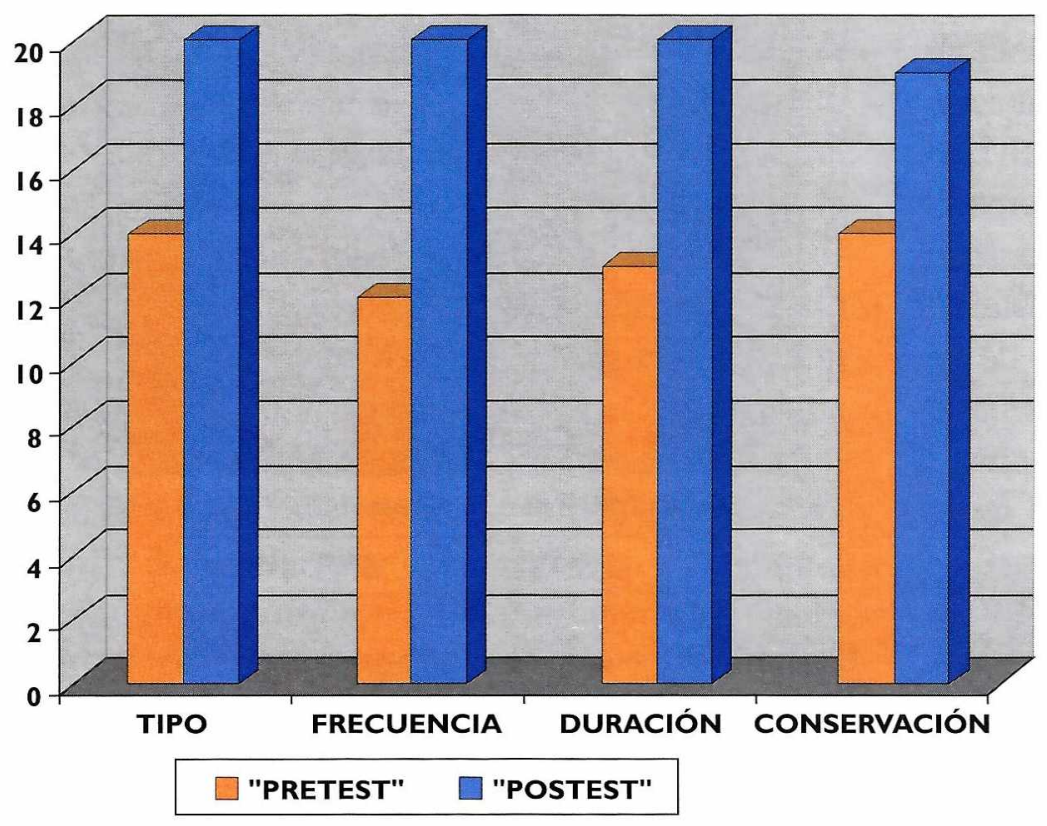

Figura 3. Distribución de frecuencias sobre los conocimientos de las madres acerca de la alimentación del niño prematuro antes y después de la intervención.

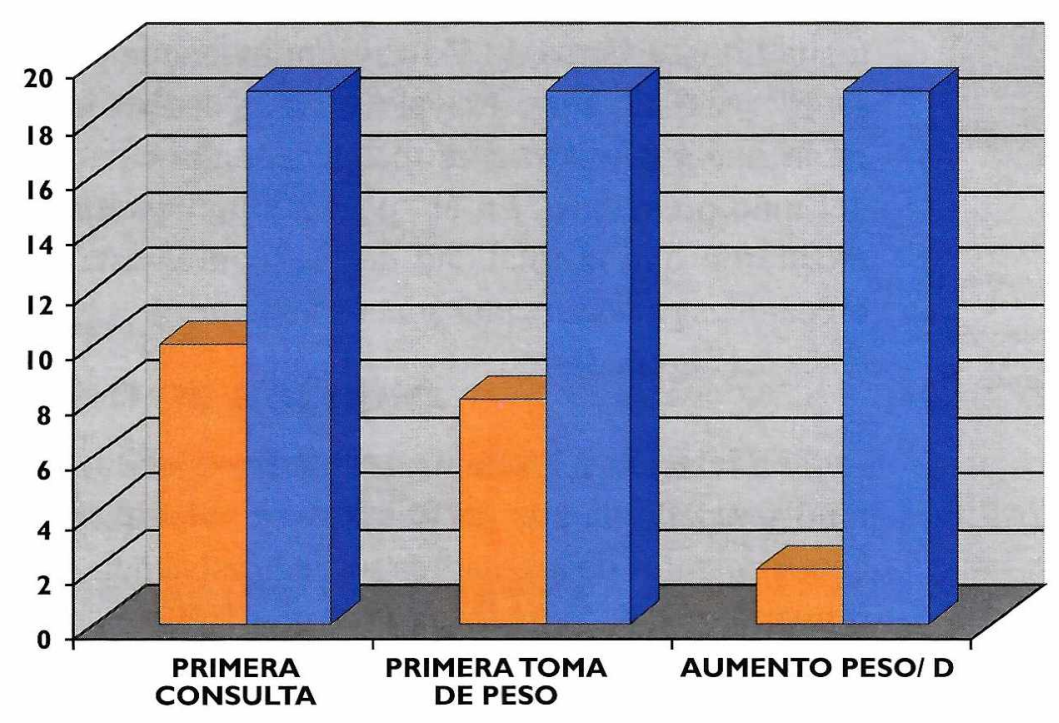

"PRETEST" $\square$ "POSTEST"

Figura 4. Distribución de frecuencias sobre los conocimientos de las madres acerca de los controles médicos antes y después de la intervención.
En cuanto a las manifestaciones de afecto que puede brindarle la madre al recién nacido se sabe que estas contribuyen para su buen desarrollo y cumplimiento de los objetivos del programa madre canguro, dentro de los resultados encontrados; en el "pretest", solo 13 madres tenían conocimientos acerca de este tema mientras que en el "postest" las 20 madres sabían cómo demostrarle afecto a sus hijos.

Luego de la recolección de los datos del "pretest" y postest" se buscó una prueba estadística que se ajustara en el momento de realizar el análisis comparativo, por lo que se utilizó una prueba estadística de diferencia de proporciones, la cual no sólo sirve para comparar dos tipos de población, sino que valida la hipótesis alterna, en este caso el nivel de significancia menor de 0,05 . Esto comprueba la hipótesis alterna en el estudio a excepción de los conocimientos en cuanto al baño del recién nacido, pues no hubo una diferencia significativa; en efecto los 2 grupos tenían conocimientos sobre este cuidado, puesto que en el "pretest" el $85 \%$ de las madres tenían conocimientos y en el "postest" el $100 \%$, lo que nos indica que porcentualmente es una diferencia mínima.

A pesar de que la muestra fue pequeña se observó que hubo influencia de la intervención, pues por ser el cuidado del recién nacido prematuro en casa de vital importancia; las madres podrán afianzar sus conocimientos y así brindarle un óptimo cuidado al niño una vez sea dado de alta de la unidad de recién nacidos. 


\section{Discusión}

La enfermera juega un papel importante en la identificación temprana de problemas de salud en los niños prematuros, que tiene un impacto significativo en su prevención. La enfermera al proporcionar soporte emocional y educativo a los padres de familia, facilita el manejo del niño, coadyuvando a perder el temor por atenderlo; debe lograr una participación activa de la familia para ofrecer un nivel de vida de calidad y un entorno cálido para el niño el cuál se integrará a la sociedad con un actuar positivo y provechoso tanto para él como en la familia. Este fue uno de los motivos que impulsó a las autoras de la presente investigación a realizar el estudio.

$\mathrm{Al}$ considerar que la diferencia entre la medición "pretest" y "post-test" fue significativa, puede afirmarse que en este grupo se presentaron cambios positivos en los conocimientos de las madres intervenidas los cuales se vieron reflejados en la tranquilidad con que las madres se acercaban a sus hijos y en el óptimo cuidado que cada una de ellas pudo brindarles. Estos resultados concuerdan con los obtenidos por Amado Yudy en $1998^{4}$ y Meneses Martha en el año $2001^{5}$ quienes mostraron que al brindar educación sobre los cuidados del recién nacido en casa se reduce el nivel de angustia de los padres, les da mayor seguridad al brindar los cuidados, fortalece el vínculo madre-hijo lo que lleva a mejorar la calidad de vida del niño prematuro.

Una de las mayores limitaciones en el desarrollo del programa, fue la duración del mismo y la dificultad de reunir dentro del tiempo planeado para la recolección de los datos el grupo de madres con los criterios de inclusión exigidos para el estudio.

\section{Referencias}

1. Eben J D. Orem. D E. Teoría del déficit de autocuidado en enfermería. En Marriner a. Modelos y teorías de enfermería. Barcelona Rol, 1989. p 101-113.

2. Ostiguin R. Teoría general del déficit de autocuidado. Buenos Aires: Manual Moderno, 2001. p. $24-25$.

3. Tolentino M.B. The use of Orem's. Selfcare Model in the NeonatalI Intensive-Care unit. J obstet Gynecol Neonatal Nurs 1990; 19 (6): 496-500.

4. Amado J. ¿Qué efectos tiene un taller educativo acerca de los cuidados del recién nacido de las madres primigestantes usuarias de ese hospital San Rafael de Facatativa (tesis) Bogotá : FUCS, 1998. pp 194-95.

5. Meneses P. Programa educativo sobre los cuidados del niño prematuro en casa para un grupo de padres que ingresan al programa madre canguro del hospital Central de la Policía en Bogotá durante el segundo semestre de 2000 y primer semestre de 2001 (tesis) Bogotá: FUCS, 2001. p 101-3.

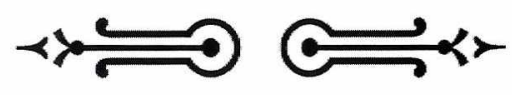

